Pacific Journal of Mathematics

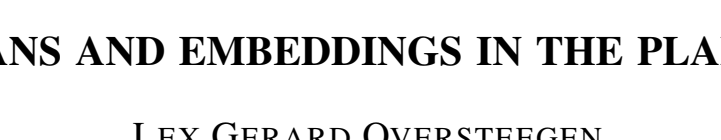




\title{
FANS AND EMBEDDINGS IN THE PLANE
}

\section{LeX G. Oversteegen}

\begin{abstract}
We prove that every fan which is locally connected at its vertex can be embedded in the plane. This gives a solution to a problem raised by J. J. Charatonik and Z. Rudy.
\end{abstract}

1. Introduction and definitions. In 1963, K. Borsuk [4] constructed a fan which is not embeddable in the plane. Hence, the question arises to characterize those fans which are embeddable in the plane. In particular, in [5] it was asked whether each contractible fan is embeddable in the plane. In an attempt to solve this problem in the negative, J. J. Charatonik and $\mathrm{Z}$. Rudy constructed a contractible fan which is locally connected at its vertex. They conjectured ([6], p. 215) that this fan is not embeddable in the plane. We show in this paper that each fan, which is locally connected at its vertex, is embeddable in the plane (see Theorem 5.2). We will also establish, for fans, several equivalences between the local connectedness at the vertex and other conditions. In a forthcoming paper [11] the author has shown that each contractible fan is locally connected at its vertex, and hence embeddable in the plane.

By a continuum we mean a compact connected metric space. A dendroid is an arc-wise connected and hereditarily unicoherent continuum. By a fan we understand a dendroid which has exactly one branch-point, and we call this branch-point the vertex of the fan. If $x, y$ are points in a dendroid $X$, then we denote by $[x, y]$ the unique arc in $X$ having $x$ and $y$ as end-points. The weak-cut order $\leqq$, with respect to a point $p$, in a dendroid $X$ is given by

$$
x \leqq y \text { if and only if }[p, x] \subset[p, y] \text {. }
$$

We denote by $I$ the unit closed interval $[0,1]$ of reals, and the symbol $B(x, \varepsilon)$ denotes the open ball of radius $\varepsilon$ about the point $x$. We use the symbol $\cong$ to denote that two spaces are homeomorphic. The symbol $R$, as used in Lemma 3.1, denotes a set of indices.

2. Embeddings in the plane. A cover $U=\left\{U_{1}, U_{2}, \cdots, U_{n}\right\}$ of a space is called an $\varepsilon$-chain if the nerve (see [8], p. 318) of $U$ is an arc and $\operatorname{diam}\left(U_{i}\right)<\varepsilon$ for $i=1,2, \cdots, n$. A continuum $X$ is said to be arc-like if for each $\varepsilon>0$ there exists an $\varepsilon$-chain covering $X$. A point $e$ of an arc-like continuum $X$ is called an end-point provided for each $\varepsilon>0$ there exists an $\varepsilon$-chain $U_{1}, U_{2}, \cdots, U_{n}$ covering $X$ such that 


$$
e \in U_{1} \mid \bigcup_{i=2}^{n} U_{i} .
$$

It is known (see [9], p. 148) that every 0-dimensional compact metric space $K$ is homeomorphic to a subset of the Cantor ternary set $C \subset[0,1]$, and hence $K$ possesses a natural order $\leqq$. We will call this ordering the induced ordering on $K$. The main result of this section is Theorem 2.2. We start with the following lemma.

Lemma 2.1. Let $X$ be a compact metric space and let $\left\{J_{\alpha}\right\}, \alpha \in A$, be the decomposition of $X$ into components. Let $\varepsilon>0$ and let $K$ be a 0-dimensional compact set in $X$, with induced ordering $\leqq$ such that:

(2) $J_{\alpha}$ is an arc-like continuum for each $\alpha \in A$,

(3) $J_{\alpha} \cap K=\left\{e_{\alpha}\right\}$, where $e_{\alpha}$ is an end-point of $J_{\alpha}$ for each $\alpha \in A$. Then there exists an open cover $U$ of $X$ such that $U$ is a finite union of disjoint $\varepsilon$-chain $V_{i}(i=1,2, \cdots, t)$, where $V_{i}=\{U(i, j)\}(j=1,2, \cdots$, $k(i))$ such that:

(4) $K \subset \mathbf{U}_{i=1}^{t} U(i, 1) \backslash \bigcup_{i=1}^{t} \bigcup_{j=2}^{k(i)} U(i, j)$,

(5) all nonadjacent elements of $U$ have positive distance,

(6) for each $i, 1 \leqq i \leqq t$, there exist $a_{i}, b_{i} \in K$ such that:

$$
K \cap U(i, 1)=\left\{x \in K \mid a_{i} \leqq x \leqq b_{i}\right\} \text {. }
$$

Proof. Denote by 0 the minimal and by 1 the maximum element of $K$. Let $g: X \rightarrow K$ be defined by $g(x)=e_{\alpha}$ if $x \in J_{\alpha}$, then $g$ is a monotone retraction. Let

(7) $x_{0}=\sup \left\{e \in K \mid\right.$ for each $e^{\prime} \leqq e$ there exists an open cover of $g^{-1}\left(\left[0, e^{\prime}\right]\right)$ satisfying the conclusion of Lemma 2.1$\}$, then $x_{0} \geqq 0$. By (2) and (3) there exists an $\varepsilon$-chain $U_{1}, U_{2}, U_{3}, \cdots, U_{k}$ in $X$ covering $g^{-1}\left(x_{0}\right)$ such that

$$
K \cap \bigcup_{j=2}^{k} U_{j}=\varnothing .
$$

Since $g^{-1}\left(x_{0}\right) \subset \bigcup_{j=1}^{k} U_{j}$ and $K$ is 0 -dimensional there exists a closed and open set $H \subset K$ such that $g^{-1}(H) \subset \bigcup_{j=1}^{k} U_{j}$. Moreover, we can choose $H$ such that

$$
H \cap K=\{x \in K \mid a \leqq x \leqq b\}
$$

for some $a$ and $b$ in $K$. If $a>0$, define $x_{1}=\sup \{x \in K \mid x<a\}$, then $x_{1} \notin U_{1}$ and $x_{1}<a$. By (7) there exists a cover $U$ of $g^{-1}\left(\left[0, x_{1}\right]\right)$ satisfying the conclusions of the lemma (if $a=0$, take $U=\varnothing$ ). Since $g^{-1}\left(\left[0, x_{1}\right]\right)$ is open in $X$ we may assume that $U U \subset g^{-1}\left(\left[0, x_{1}\right]\right)$. Hence

$$
U \cup\left\{U_{j} \cap g^{-1}(H) \mid j=1,2, \cdots, k\right\}
$$


is a cover of $g^{-1}([0, b])$ satisfying the conclusion of the lemma. It follows the definition of $x_{0}$ that $x_{0}=b$.

If $x_{0}=b=1$, we are done, whence suppose $x_{0}<1$ and let $x_{2}=$ inf $\left\{x \in K \mid x>x_{0}\right\}$. By repeating the argument above, replacing $x_{0}$ by $x_{2}$, one can show that $g^{-1}\left(\left[0, x_{2}\right]\right)$ can be covered with a cover satisfying the conclusion of the lemma, contrary to (7), since $x_{2}>x_{0}$.

We will call a cover $U$ that satisfies the conclusion of Lemma 2.1 an $\varepsilon$-cover of $X$.

THEOREM 2.2. Let $X$ be a compact metric space and $K$ a closed subset of $X$. Let $\left\{J_{\alpha}\right\}, \alpha \in A$, be the decomposition of $X$ into components such that:

(8) $J_{\alpha} \cap K=\{e\}$, where $e$ is an end-point of $J_{\alpha}$ for each $\alpha \in A$,

(9) $J_{\alpha}$ is an arc-like continuum for each $\alpha \in A$. Then there exists an embedding $h: X \rightarrow I^{2}$ such that $h(K)=h(X) \cap l$, where $l=$ $\left\{(x, y) \in I^{2} \mid y=0\right\}$.

Proof. Notice that by (8) $K$ is 0-dimensional. By Lemma 2.1, there exists for each $\varepsilon>0$ an $\varepsilon$-cover of $X$. Let $U_{1}$ be a $1 / 2$-cover of $X$ and $\eta>0$ such that $\eta$ is the minimum distance between two nonintersecting elements of $U_{1}$. By induction we construct a sequence of covers $U_{1}, U_{2}, \cdots$ of $X$ such that $U_{n}$ refines $U_{n-1}, U_{n}$ is a $(1 / 2)^{n_{-}}$ cover, no sub-chain of less than nine links of $U_{n}$ connects two nonintersecting elements of $U_{n-1}$.

Given a cover $U$ of $X$, satisfying the conclusion of Lemma 2.1, we label the chains $V_{1}, V_{2}, \cdots, V_{t}$ of $U$ such that inf $\left\{x \mid x \in K \cap V_{i}\right\}<$ $\inf \left\{x \mid x \in K \cap V_{i}\right\}$ if $i<j$, and the links of the chain $V_{i}=\{U(i, 1)$, $U(i, 2), \cdots, U(i, k(i))\}$ such that $K \cap V_{i} \subset U(i, 1)$. If $U$ and $U^{*}$ are both covers of $X$, satisfying the conclusion of Lemma 2.1, then we say that $U$ follows the pattern $\left\{\left(a_{1}, b_{1}\right),\left(a_{1}, b_{2}\right), \cdots,\left(a_{1}, b_{k(1)}\right), \cdots\right.$, $\left.\left(a_{t}, b_{k(t)}\right)\right\}$ in $U^{*}$ if the $j$ th link of the $i$ th chain of $U$ is contained in the $b_{j}$ th link of the $a_{i}$ th chain of $U^{*}$ (i.e., $U(i, j) \subset U^{*}\left(a_{i}, b_{j}\right)$.

There exist in $I^{2}$ a sequence of open sets $D_{1}, D_{2}, \cdots$ such that $D_{n}$ is a finite union of $(1 / 2)^{n}$-chains whose elements are interiors of rectangles, and such that $D_{n}$ follows a pattern in $D_{n-1}$ that $U_{n}$ follows in $U_{n-1}$, each element of $D_{n-1}$ contains the closure of an element of $D_{n}$, while the closure of each element of $D_{n}$ lies in an element of $D_{n-1}$ and the first link of each chain of $D_{n}$ intersects $l$ in a nondegenerate interval, while the closure of all other elements of $D_{n}$ are contained in $\left.I^{2}\right\rangle(n=1,2 \cdots)$.

The existence of the open sets $D_{n}$ satisfying the above follows from an argument similar to one used by R. H. Bing (see [3], p. 654), 
the only difference being that in each cover $D_{n-1}$ we insert, in the next step, finitely many, instead of one, new chains and we require the first link of each chain of $D_{n}$ to intersect $l$ in a nondegenerate interval, while the closures of all other elements of $D_{n}$ are contained in $\left.I^{2}\right\rangle l$.

The latter facts can be established by dividing each chain of $D_{n-1}$ into finitely many "strips" in each of which we insert, in the next step, a new chain in such a way that we always insert new links on a predescribed "side" of already chosen previous links.

It follows from Theorem 11 of [2] that $X$ is homeomorphic with the continuum $Y=D_{1}^{*} \cap D_{2}^{*} \cap \cdots$, where $D_{n}^{*}$ denotes the union of the elements of $D_{n}$ and moreover it follows from the choice of $D_{n}$ that $Y$ satisfies the conclusion of Theorem 2.2, and the proof is complete.

3. Fans locally connected at the vertex. A fan $X$ has property $P^{1}$, if for each sequence of points $\left\{x_{i}\right\}$ in $X(i=1,2, \cdots)$ converging to the vertex $v$ of $X$ we have

$$
L s\left[v, x_{i}\right]=\{v\} .
$$

THEOREM 3.1. Let $X$ be a fan with vertex $v$ and

(2) $X=\bigcup_{r \in R}\left\{J_{r} \mid J_{r} \cong[0,1]\right.$ for each $r \in R$ and $J_{r_{1}} \cap J_{r_{2}}=\{v\}$ if $\left.r_{1} \neq r_{2} \in R\right\}$,

then the following are equivalent:

(3) $X$ has property $P$,

(4) for each $\varepsilon>0$, there exists a connected open neighborhood $U$ of $v$ such that $\operatorname{diam}(U) \leqq \varepsilon$ and $\operatorname{Bd}(U) \cap J_{r}$ is connected for every $r \in R$,

(5) $X$ is locally connected at $v$.

Proof. (3) $\rightarrow(4)$. Let $\varepsilon>0$ be given and let $\leqq$ be the weakcut order of $X$ with respect to $v$. Define $V=B(v, e)$,

$$
\begin{gathered}
x(r)=\inf \left\{x \in X \mid x \in J_{r} \cap \mathrm{Bd}(V)\right\} \text { if } J_{r} \cap \mathrm{Bd}(V) \neq \varnothing, \\
Q_{r}= \begin{cases}\left\{y \in J_{r} \mid y \geqq x(r)\right\} & \text { if } J_{r} \cap \mathrm{Bd}(V) \neq \varnothing \\
\varnothing & \text { otherwise }\end{cases}
\end{gathered}
$$

and $Q=\bigcup_{r \in R} Q_{r}$. It follows that $v \notin \bar{Q}$, since if $\left\{v_{i}\right\}$ is a sequence in $Q$ converging to $v$, then $v_{i} \geqq x\left(r_{i}\right)$ for some $r_{i} \in R$, and hence $L s\left[v, v_{i}\right] \cap \mathrm{Bd}(V) \neq \varnothing$, contrary to (3).

Let $U=X \mid \bar{Q}$, then $U$ is an open neighborhood of $v$ and $\operatorname{diam}(U) \leqq$

1 It follows from the definition that property $P$ is related to the notion of a $Q$ point or a $P$-point (cf. [1] and [7], respectively). 
$\operatorname{diam}(V)=\varepsilon$. We will show that $U$ satisfies all conditions of (4). We claim that

(7) if $z \in U$ and $x<z$, then $x \in U$, or, equivalently, if $x \in \bar{Q}$ and $z \geqq x$, then $z \in \bar{Q}$.

To this end, suppose that (7) is false. Hence $x \in \bar{Q}$, let $\left\{x_{i}\right\}$ be a sequence in $Q$ converging to $x$. Then $x_{i} \geqq x\left(r_{i}\right) \in \mathrm{Bd}(V)$ for some $r_{i} \in R(i=1,2, \cdots)$. We may assume that the sequence $\left\{x\left(r_{i}\right)\right\}$ converges to a point $x_{0} \in J_{r_{0}} \cap \mathrm{Bd}(V)$ for some $r_{0} \in R$.

By ([9], p. 171), $L s\left[x_{i}, x\left(r_{i}\right)\right]$ is a continuum and since $\left[x_{i}, x\left(r_{i}\right)\right] \subset$ $Q(i=1,2, \cdots)$ we have $L s\left[x_{i}, x\left(r_{i}\right)\right] \subset \bar{Q} \subset X \backslash\{v\}$. Moreover, since $X$ is hereditarily unicoherent, it follows that $\left[x, x_{0}\right] \subset L s\left[x_{i}, x\left(r_{i}\right)\right] \subset \bar{Q} \subset$ $X \backslash\{v\}$ and we consider two cases as follows:

Case 1. $z \in\left[x, x_{0}\right]$. Then $z \in \bar{Q}$.

Case 2. $z \notin\left[x, x_{0}\right]$. Then, since $z>x, z>\max \left\{x, x_{0}\right\}$ and consequently $z>x_{0} \geqq x\left(r_{0}\right)$. Hence $z \in Q$ by (6) and the definition of $Q$.

In both case we conclude that $z \in \bar{Q}$, contrary to the assumptions in (7) and the proof of (7) is complete. It follows from (7) that $U$ is connected. In order to show that $J_{r} \cap \mathrm{Bd}(U)$ is connected for each $r \in R$, we will show that if $x, y \in J_{r} \cap \operatorname{Bd}(U)$, say $x<y$, and $z \in[x, y]$, then $z \in J_{r} \cap \operatorname{Bd}(U)$.

Since $x \in J_{r} \cap \operatorname{Bd}(U)=J_{r} \cap \bar{U} \cap \bar{Q}$ and $z>x$, it follows from (7) that $z \in \bar{Q}$. Moreover, since $y \in \bar{U}$, there exists a sequence $\left\{y_{i}\right\}$ in $U$ converging to $y$. Since $L s\left[v, y_{i}\right]$ is a continuum ([9], $p$. 171), containing both $y$ and $v$ and $X$ is hereditarily unicoherent, it follows that $[v, y] \subset L s\left[v, y_{i}\right]$. As $z \in[v, y]$, we may assume that there exists a sequence $\left\{z_{i}\right\}$, where $z_{i} \in\left[v, y_{i}\right]$, converging to $z$. By (7), $z_{i} \in U$ and whence $z \in \bar{U}$. Obviously $z \in J_{r}$ and we conclude $z \in J_{r} \cap \bar{U} \cap \bar{Q}=$ $J_{r} \cap \operatorname{Bd}(U)$.

$(4) \rightarrow(5)$ : Trivial.

$(5) \rightarrow(3)$ : Suppose $X$ does not have property $P$. Let $\left\{x_{i}\right\}$ be a sequence of points in $X$ converging to $v$ such that $L s\left[v, x_{i}\right]=K \neq\{v\}$.

Let $\varepsilon>0$ be such that $\operatorname{diam}(K)>3 \varepsilon$ and let $U$ be a connected neighborhood of $v$ such that $\operatorname{diam}(U)<\varepsilon$. Then there exists an index $i>0$ such that $x_{i} \in U$ and $\left[v, x_{i}\right] \cap[X \backslash B(v, 2 \varepsilon)] \neq \varnothing$. But then $\bar{U}$ and $\left[v, x_{i}\right]$ are two continua in $X$ whose intersection is not connected, contradicting the fact that $X$ is hereditarily unicoherent, and the proof is complete.

4. Decompositions of fans. We say that a space $X$ is a $(q=c)$ space if, in $X$, every quasi-component is connected. In other words, for $(q=c)$-spaces the quasi-components and the components coincide. We will show that if a fan is locally connected at the vertex $v$ of 
$X$, then $X \backslash\{v\}$ is a $(q=c)$-space.

THEOREM 4.1. Let $X$ be a fan which is locally connected at the vertex $v$ of $X$ and

$$
\begin{aligned}
& X=\bigcup_{r \in R}\left\{J_{r} \mid J_{r} \cong[0,1] \text { for each } r \in R \text { and } J_{r_{1}} \cap J_{r_{2}}=\{v\}\right. \\
& \text { if } \left.r_{1} \neq r_{2} \in R\right\} .
\end{aligned}
$$

Then $X \backslash\{v\}$ is a $(q=c)$-space and $\left\{J_{r} \backslash\{v\}\right\}, r \in R$, is the decomposition of $X \backslash\{v\}$ into quasi-components.

Proof. It is sufficient to show that if $r_{0} \neq r_{1} \in R$, then there exists a closed and open set $G \subset X \backslash\{v\}$ such that

$$
J_{r_{0}} \backslash\{v\} \subset G \subset X \backslash J_{r_{1}} .
$$

By Theorem 3.1 there exists for each $n(n=1,2, \cdots)$ a neighborhood $U_{n}$ of $v$ such that $\operatorname{diam}\left(U_{n}\right)<1 / n, \bar{U}_{n+1} \subset U_{n}$ and $\operatorname{Bd}\left(U_{n}\right) \cap J_{r}$ is connected for each $r \in R$. We may assume that $J_{r_{0}} \cap \operatorname{Bd}\left(U_{1}\right) \neq$ $\varnothing \neq J_{r_{1}} \cap \mathrm{Bd}\left(U_{1}\right)$. Let $R_{n}=\left\{r \in R \mid \operatorname{Bd}\left(U_{n}\right) \cap J_{r} \neq \varnothing\right\}(n=1,2, \cdots)$, then $R_{n} \subset R_{n+1}$ and $\bigcup_{n=1}^{\infty} R_{n}=R$.

Let $Y$ be the space obtained from $\mathrm{Bd}\left(U_{1}\right)$ by identifying all components of $\operatorname{Bd}\left(U_{1}\right)$ to a point and let $f: \operatorname{Bd}\left(U_{1}\right) \rightarrow Y$ be the natural projection. It follows ([9], p. 148) that $\operatorname{dim} Y=0$. Since

$$
f\left(J_{r_{0}} \cap \operatorname{Bd}\left(U_{1}\right)\right) \neq f\left(J_{r_{1}} \cap \operatorname{Bd}\left(U_{1}\right)\right),
$$

there exists a closed and open set $H_{1}^{*}$ in $Y$ such that

$$
f\left(J_{r_{0}} \cap \operatorname{Bd}\left(U_{1}\right)\right) \subset H_{1}^{*} \subset Y \backslash f\left(J_{r_{1}} \cap \operatorname{Bd}\left(U_{1}\right)\right) .
$$

Let $H_{1}=f^{-1}\left(H_{1}^{*}\right)$, then $H_{1}$ is a closed and open set in $\operatorname{Bd}\left(U_{1}\right)$. Define $A_{1}=\left\{r \in R_{1} \mid J_{r} \cap H_{1} \neq \varnothing\right\}$ and $B_{1}=\left\{r \in R_{1} \mid J_{r} \cap H_{1}=\varnothing\right\}$, then $A_{1} \cap B_{1}=\varnothing$ and $A_{1} \cup B_{1}=R_{1}$. Moreover, since $H_{1}$ is closed and open in $\operatorname{Bd}\left(U_{1}\right)$, we have that

$$
P_{1}=\bigcup_{r \in A_{1}}\left\{J_{r} \mid\{v\}\right\} \text { and } Q_{1}=\bigcup_{r \in B_{1}}\left\{J_{r} \mid\{v\}\right\}
$$

are disjoint and closed subsets of $X \backslash\{v\}$.

By induction we will construct sets $A_{n}$ and $B_{n}$ such that

$$
A_{n-1} \subset A_{n}, B_{n-1} \subset B_{n}, A_{n} \cap B_{n}=\varnothing \text { and } A_{n} \cup B_{n}=R_{n}
$$

and if $P_{n}=\bigcup_{r \in A_{n}}\left\{J_{r}\right\}$ and $Q_{n}=\bigcup_{r \in B_{n}}\left\{J_{r}\right\}$ then $P_{n}$ and $Q_{n}$ are disjoint and closed subsets of $X \backslash\{v\}(n=1,2, \cdots)$.

Suppose $A_{n-1}$ and $B_{n-1}$ have been constructed. Since $P_{n-1} \cap$ $\operatorname{Bd}\left(U_{n}\right)$ and $Q_{n-1} \cap \operatorname{Bd}\left(U_{n}\right)$ are disjoint closed subsets of $\operatorname{Bd}\left(U_{n}\right)$ and 
$J_{r} \cap \operatorname{Bd}\left(U_{n}\right)$ is connected for each $r \in R$, it follows as above, replacing $U_{1}, J_{r_{0}} \cap \operatorname{Bd}\left(U_{1}\right)$ and $J_{r_{1}} \cap \operatorname{Bd}\left(U_{1}\right)$ by $U_{n}, P_{n-1} \cap \operatorname{Bd}\left(U_{n}\right)$ and $Q_{n-1} \cap \operatorname{Bd}\left(U_{n}\right)$ respectively, that there exists a closed and open subset $H_{n}$ of $\operatorname{Bd}\left(U_{n}\right)$ such that

$$
P_{n-1} \cap \operatorname{Bd}\left(U_{n}\right) \subset H_{n} \subset \operatorname{Bd}\left(U_{n}\right) \backslash Q_{n-1} .
$$

Let $A_{n}=\left\{r \in R_{n} \mid J_{r} \cap H_{n} \neq \varnothing\right\}$ and $B_{n}=\left\{r \in R_{n} \mid J_{r} \cap H_{n}=\varnothing\right\}$, then $A_{n}$ and $B_{n}$ satisfy (2).

Let $A=\bigcup_{n=1}^{\infty} A_{n}$ and $B=\bigcup_{n=1}^{\infty} B_{n}$, then $A \cup B=R$ and $A \cap B=$ $\varnothing$. Let $G=\bigcup_{r \in A}\left\{J_{r} \backslash\{v\}\right\}$ and $G_{n}=\bigcup_{r \in A_{n}}\left\{J_{r} \backslash \bar{U}_{n}\right\}$. Since $G_{n}$ is open in $X$ and $G=\bigcup_{n=1} G_{n}$, it follows that $G$ is open in $X$. Similarly $X \backslash(G \cup\{v\})=\bigcup_{r \in B}\left\{J_{r} \backslash\{v\}\right\}$ is open in $X$. Hence $G$ is both open and closed in $X \backslash\{v\}$ and, since $r_{0} \in A_{1}$ and $r_{1} \in B_{1}$, (1) is proved.

5. Property $P$ and embeddings in the plane. The main result of this section is Theorem 5.2 where we prove that if a fan is locally connected at its vertex, then it can be embedded in the plane. This result gives a solution to problem 1015 of [6].

Since every fan is hereditarily decomposable and hence 1-dimensional ([9], p. 206), we can consider every fan as a subspace of $I^{3}$. We start with the following lemma.

LEMma 5.1. Let $X$ be a fan, with vertex $v$ and

$$
\begin{aligned}
& X=\bigcup_{r \in R}\left\{J_{r} \mid J_{r} \cong[0,1] \text { for each } r \in R \text { and } J_{r_{1}} \cap J_{r_{2}}=\{v\}\right. \\
& \text { if } \left.r_{1} \neq r_{2} \in R\right\}
\end{aligned}
$$

such that $\left\{J_{r} \backslash\{v\}\right\}, r \in R$, is the decomposition of $X \backslash\{v\}$ into quasicomponents, then there exists an embedding $f: X \backslash\{v\} \rightarrow C \times I^{3}$ such that each quasi-component of $X \backslash\{v\}$ is contained in $\{c\} \times I^{3}$ for some $c \in C$, and

$$
\overline{f(X \backslash\{v\}}) \backslash f(X \backslash\{v\}) \subset C \times\{v\},
$$

where $C \subset[0,1]$ denotes the Cantor ternary set.

Proof. We may assume that $X \subset I^{3}$. By ([9], p. 148), there exists a continuous function $g: X \backslash\{v\} \rightarrow C$ such that the quasi-components of $X \backslash\{v\}$ coincide with the point-inverses of $g$. Then the function $f: X \backslash\{v\} \rightarrow C \times I^{3}$ defined by $f(x)=(g(x), x)$ is an embedding. Only (1) remains to be shown. Let

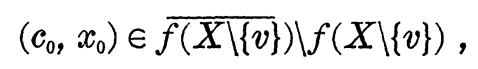

and let $\left\{\left(c_{i}, x_{i}\right)\right\}(i=1,2, \cdots)$ be a sequence of points in $f(X \backslash\{v\})$ converging to $\left(c_{0}, x_{0}\right)$. We may assume that the sequence $\left\{x_{i}\right\}$ in $X$, 
where $x_{i}=f^{-1}\left(\left(c_{i}, x_{i}\right)\right)$, converges to a point $y \in X$. We consider two cases as follows:

Case 1. $y \neq v$. Then the sequence $\left\{f\left(x_{i}\right)\right\}$, where $f\left(x_{i}\right)=\left(c_{i}, x_{i}\right)$, converges to $f(y)$. Hence $f(y)=\left(c_{0}, x_{0}\right)$, contrary to (2).

Case 2. $y=v$. Then $x_{0}=v$ and whence (1) holds.

These two cases complete the proof of the lemma.

THEOREM 5.2. Let $X$ be a fan which is locally connected at the vertex $v$ of $X$, then $X$ is embeddable in the plane.

Proof. Let

$$
\begin{aligned}
& X=\bigcup_{r \in R}\left\{J_{r} \mid J_{r} \cong[0,1] \text { for each } r \in R \text { and } J_{r_{1}} \cap J_{r_{2}}=\{v\}\right. \\
& \left.\quad \text { if } r_{1} \neq r_{2} \in R\right\} .
\end{aligned}
$$

It follows from 4.1 that $\left\{J_{r} \backslash\{v\}\right\}, r \in R$, is the decomposition of $X \backslash\{v\}$ into quasi-components. Hence by Lemma 5.1 there exists an embedding $f: X \backslash\{v\} \rightarrow C \times I^{3}$ such that each quasi-component of $X \backslash\{v\}$ is contained in $\{c\} \times I^{3}$ for some $c \in C$ and

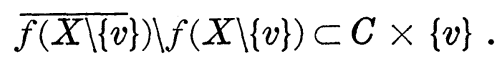

It follows that $\overline{f(X \backslash\{v\})}$ satisfies all conditions of Theorem 2.2, where $K=\overline{f(X \backslash\{v\})} \cap(C \times\{v\})$. Hence there exists an embedding $h: \overline{f(X \backslash\{v\})} \rightarrow$ $I^{2}$ such that $\left.h(K)=h(\overline{f(X \backslash\{v\}})\right) \cap l$, where $l=\left\{(x, y) \in I^{2} \mid y=0\right\}$. Let $\pi: I^{2} \rightarrow I^{2} / l$ be the natural projection. It follows (see [9], p. 533) that $I^{2} \cong I^{2} / l$ and whence the mapping $g: X \rightarrow I^{2} / l$ defined by

$$
g(x)= \begin{cases}\pi \circ h \circ f(x) & \text { if } x \neq v, \\ \pi(l) & \text { if } x=v\end{cases}
$$

is the required embedding.

REMARK. J. J. Charatonik and Z. Rudy constructed a fan $X$ which is locally connected at its vertex (see [6], p. 215). They conjectured that this fan is not embeddable in the plane. The above theorem disproves their conjecture and gives a solution to problem 1015 of [6].

Acknowledgments. The author wishes to thank Prof. A. Lelek for some valuable suggestions. This research was supported by a Graduate Fellowship from Wayne State University. 


\section{REFERENCES}

1. Ralph B. Bennett, On some classes of non-contractible dendroids, Institute of the Polish Academy of Sciences, Mimeographed Paper (1972) (unpublished).

2. R. H. Bing, A homogeneous indecomposable plane continuum, Duke J. of Math., 15 (1948), 729-742.

3. — Snake-like continua, ibidem 18 (1950), 653-663.

4. K. Borsuk, A countable broom which cannot be imbedded in the plane, Colloq. Math., 10 (1963), 233-236.

5. J. J. Charatonik and C. A. Eberhart, On contractible dendroids, Colloq. Math., 25 (1972), 89-98.

6. J. J. Charatonik and Z. Rudy, Remarks on non-planable dendroids, Colloq. Math., 37 (1977), 205-216.

7. B. G. Graham, On contractible fans, Doctoral dissertation, University of California, Riverside, California (1977).

8. K. Kuratowski, Topology Vol. I, Academic Press (1966).

9. — Topology Vol. II, ibidem, 1968.

10. Lex G. Oversteegen, Properties of contractible fans, Doctoral dissertation, Wayne State University, Detroit, Michigan, 1978.

11. - Every contractible fan is locally connected at its vertex, Trans. Amer. Math. Soc., (to appear).

Received June 12, 1978 and in revised form January 8, 1979. This paper is part of the author's doctoral dissertation, completed at Wayne State University, Detroit, Michigan.

UNIVERSITY OF HOUSTON

HousTon, TX 77004 



\title{
PACIFIC JOURNAL OF MATHEMATICS
}

\section{EDITORS}

DONALD BABBITT (Managing Editor)

University of California

Los Angeles, CA 90024

\section{HUGO RossI}

University of Utah

Salt Lake City, UT 84112

C. C. MOORE and ANDREW OGG

University of California

Berkeley, CA 94720

\section{J. DUGUNDJI}

Department of Mathematics University of Southern California Los Angeles, CA 90007

R. FINN and J. Milgram

Stanford University Stanford, CA 94305

\section{ASSOCIATE EDITORS}
E. F. BECKENBACH
B. H. NeUmanN
F. WOLF
K. YosHIDA

\section{SUPPORTING INSTITUTIONS}

\author{
UNIVERSITY OF BRITISH COLUMBIA \\ CALIFORNIA INSTITUTE OF TECHNOLOGY \\ UNIVERSITY OF CALIFORNIA \\ MONTANA STATE UNIVERSITY \\ UNIVERSITY OF NEVADA, RENO \\ NEW MEXICO STATE UNIVERSITY \\ OREGON STATE UNIVERSITY \\ UNIVERSITY OF OREGON
}

\author{
UNIVERSITY OF SOUTHERN CALIFORNIA \\ STANFORD UNIVERSITY \\ UNIVERSITY OF HAWAII \\ UNIVERSITY OF TOKYO \\ UNIVERSITY OF UTAH \\ WASHINGTON STATE UNIVERSITY \\ UNIVERSITY OF WASHINGTON
}

The Supporting Institutions listed above contribute to the cost of publication of this Journal, but they are not owners or publishers and have no responsibility for its content or policies.

Mathematical papers intended for publication in the Pacific Journal of Mathematics should be in typed form or offset-reproduced, (not dittoed), double spaced with large margins. Please do not use built up fractions in the text of the manuscript. However, you may use them in the displayed equations. Underline Greek letters in red, German in green, and script in blue. The first paragraph or two must be capable of being used separately as a synopsis of the entire paper. Please propose a heading for the odd numbered pages of less than 35 characters. Manuscripts, in triplicate, may be sent to any one of the editors. Please classify according to the scheme of Math. Reviews, Index to Vol. 39. Supply name and address of author to whom proofs should be sent. All other communications should be addressed to the managing editor, or Elaine Barth, University of California, Los Angeles, California, 90024.

50 reprints to each author are provided free for each article, only if page charges have been substantially paid. Additional copies may be obtained at cost in multiples of 50 .

The Pacific Journal of Mathematics is issued monthly as of January 1966. Regular subscription rate: $\$ 84.00$ a year (6 Vols., 12 issues). Special rate: $\$ 42.00$ a year to individual members of supporting institutions.

Subscriptions, orders for numbers issued in the last three calendar years, and changes of address should be sent to Pacific Journal of Mathematics, P.O. Box 969, Carmel Valley, CA 93924, U.S.A. Older back numbers obtainable from Kraus Periodicals Co., Route 100, Millwood, NY 10546.

PUBLISHED BY PACIFIC JOURNAL OF MATHEMATICS, A NON-PROFIT CORPORATION

Printed at Kokusai Bunken Insatsusha (International Academic Printing Co., Ltd.). 8-8, 3-chome, Takadanobaba, Shinjuku-ku, Tokyo 160, Japan.

Copyright (C) 1979 by Pacific Journal of Mathematics Manufactured and first issued in Japan 


\section{Pacific Journal of Mathematics}

\section{Vol. 83, No. $2 \quad$ April, 1979}

Patrick Robert Ahern, On a theorem of Hayman concerning the derivative of a

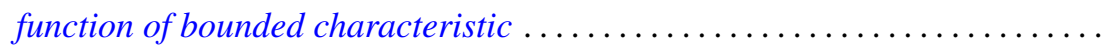

Walter Allegretto, Finiteness of lower spectra of a class of higher order elliptic

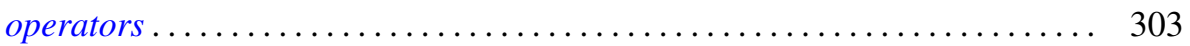

Leonard Asimow, Superharmonic interpolation in subspaces of $C_{c}(X) \ldots \ldots 11$

Steven F. Bellenot, An anti-open mapping theorem for Fréchet spaces . . . . . . . 325

B. J. Day, Locale geometry. . . . . . . . . . . . . . . . . . . . . . . . . . 333

John Erik Fornaess and Steven Krantz, Continuously varying peaking

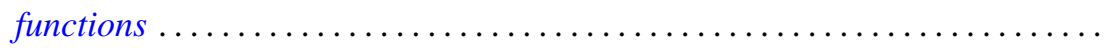

Joseph Leonide Gerver, Long walks in the plane with few collinear points ......

Joseph Leonide Gerver and Lawrence Thom Ramsey, On certain sequences of

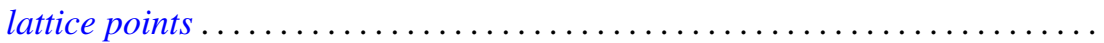

John R. Graef, Yuichi Kitamura, Takaŝi Kusano, Hiroshi Onose and Paul Winton

Spikes, On the nonoscillation of perturbed functional-differential

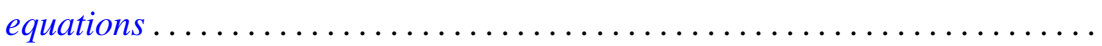

James A. Huckaba and James M. Keller, Annihilation of ideals in commutative

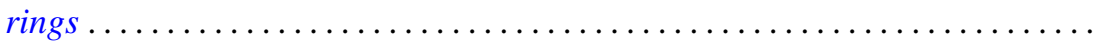

Anzelm Iwanik, Norm attaining operators on Lebesgue spaces . . . . . . . . . . .

Surjit Singh Khurana, Pointwise compactness and measurability . . . .......... 387

Charles Philip Lanski, Commutation with skew elements in rings with

involution.

Hugh Bardeen Maynard, A Radon-Nikodým theorem for finitely additive bounded

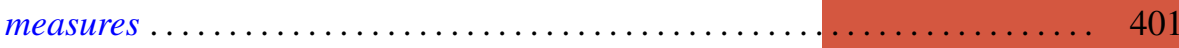

Kevin Mor McCrimmon, Peirce ideals in Jordan triple systems ..

Sam Bernard Nadler, Jr., Joseph E. Quinn and N. Stavrakas, Hyperspaces of compact convex sets.

Ken Nakamula, An explicit formula for the fundamental units of a real pure

sextic number field and its Galois closure ............

Vassili Nestoridis, Inner functions invariant connected components . .

Vladimir I. Oliker, On compact submanifolds with nondegenerate parallel

normal vector fields.

Lex Gerard Oversteegen, Fans and embeddings in the plane.

Shlomo Reisner, On Banach spaces having the property G.L

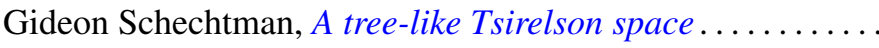

Helga Schirmer, Fix-finite homotopies . . . . . . . . . . . .

Jeffrey D. Vaaler, A geometric inequality with applications to linear forms . .

William Jennings Wickless, $T$ as an $\mathscr{G}$ submodule of $G$.....

Kenneth S. Williams, The class number of $Q(\sqrt{-p})$ modulo 4 , for $p \equiv 3$ (

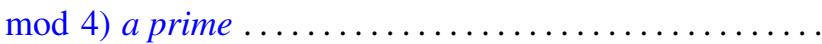

